\title{
Detection of initial subsurface defects in rotor blade leading edges of wind turbines by means of active thermography
}

\author{
Friederike Jensen ${ }^{1}$, Michael Sorg ${ }^{1}$, Andreas Fischer ${ }^{1}$ \\ 1 University of Bremen, Bremen Institute for Metrology, Automation and Quality Science \\ Linzer Str. 13, 28359, Bremen, Germany \\ Correspondence: f.jensen@bimaq.de
}

\section{Summary:}

The surface condition of the leading edge of rotor blades has a significant influence on the lifetime and performance of a wind turbine. Initial subsurface defects like air traps can lead to a premature surface damage by erosion, and a delayed defect detection results in high maintenance and repair costs. Active thermography is proposed as a non-destructive and in situ measuring method to visualize damage patterns on the rotor blade leading edges and to make initial subsurface defects visible. In particular, it enables the investigation of the damage development for mechanical impacts such as rain drops.

Keywords: active thermography, edge zone analysis, leading edge erosion, subsurface defects, composite materials

\section{Introduction}

The leading edge of a rotor blade is particularly exposed to mechanical and environmental stress, such as in rain, where the drops hit the blade at an impact speed of over $300 \mathrm{~km} / \mathrm{h}$. The impact of the raindrops gradually removes the coated surface and parts of the underlying glass fiber composite material [1]. The impact of the rotor blade damage on the life and performance of a wind turbine is considerable, and repairing such a damage is complicated and expensive. Figure 1 shows a schematic representation of the leading edge structure of eroded rotor blades.

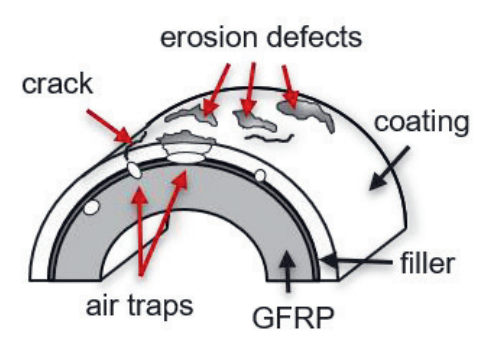

Fig. 1. Schematic sketch of a coated GFRP test specimen with initial defects (air traps) and surface defects

Studies suggest that initial subsurface defects such as pores in the interface between the coating and glass-fiber reinforced plastic (GFRP) lead to premature erosion [2]. The size of the damage-promoting subsurface defects ranges from millimeters to centimeters and remain hidden during visual inspection. Early detection and evaluation of erosion surface damage and subsurface defects, before a major damage up to and including total failure of the rotor blade occurs, leads to a reduction in maintenance and repair costs. However, the effect of certain defect properties such as the defect size on the premature damage is still unclear. Therefore it is necessary to develop a non-destructive and contactless measuring technique to visualize subsurface inhomogeneities.

\section{Measurement approach}

Long pulse thermography is selected as the measuring method for the contactless, non-destructive examination of erosion damage and subsurface defects [3], since, in contrast to computer tomography, it can also be used in situ. In active thermography, the test specimen is first heated by an energy source. In the subsequent cooling phase, the different temperature distribution at the surface of the test specimen is recorded by an infrared camera (IR) [4]. Thereafter, the thermograms are processed to visually highlight and, thus, to detect material inhomogeneities. The defects visibility is assessable by means of the contrast to noise ratio (CNR) [5].

\section{Experimental setup}

For the thermographic investigations, a test rig is set up as shown in Figure 2. The test specimen, which resembles the leading edge of a rotor blade, is fixed in a kinematic system. Using a camera of the type Vario Cam hr head (resolution: 640 x $480 \mathrm{px}$, wavelength range: $7.5-14$ $\mu \mathrm{m})$ from the company InfraTec with a $30 \mathrm{~mm}$ lens, an image of the emitted infrared light from 
the test specimen surface is obtained. Furthermore, an excitation unit, consisting of two $1 \mathrm{~kW}$ halogen lamps, is applied under and above the camera to heat the test specimen. The kinematic system as well as the IR camera is connected to a computer to position the test specimen and to evaluate the acquired image data with the software Irbis 3 from the company InfraTec. For the examination the test specimen is excited for $10 \mathrm{~s}$. The recording time starts at the end of the excitation and is set to $30 \mathrm{~s}$ at a recording frequency of $25 \mathrm{~Hz}$. Here, the first image of the raw data is considered and processed with low pass filters to identify subsurface defects.

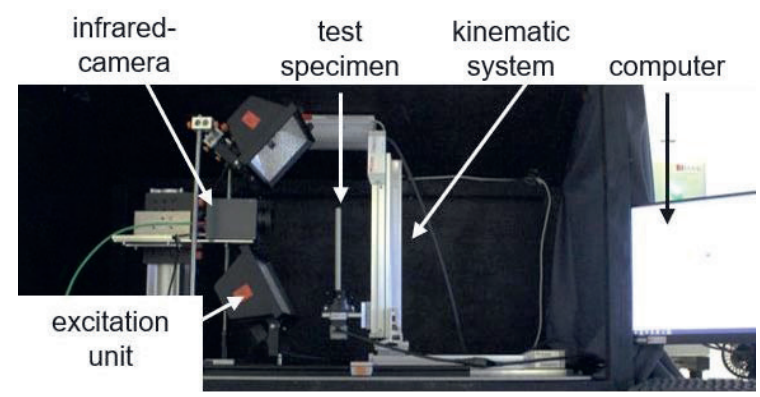

Fig. 2. Experimental setup for the thermographic examination of test specimens

\section{Results}

The measurements are carried out on a test specimen consisting of a hand-laminated GFRP half pipe which is covered with a coating system including a filler and a gel coating, both consisting of polyurethane. Polystyrene balls of an average diameter of $1 \mathrm{~mm}$ are incorporated into the coating system, which are intended to imitate air traps created during production. Figure 3 shows an image of the test specimen with its associated thermogram. Due to the prevailing temperature difference, which is caused during the cooling process by material-dependent heat storage capacities and the resulting different heat transfers, the small polystyrene balls are detected. They are recognizable as bright points in the thermograms, but remain hidden in the photograph. The CNR indicates the level of contrast between a defect and its surroundings. A high CNR stands for a high detection capability. Exemplary in the first recording, the CNR ranges between 22 and 108.

\section{Conclusion and Outlook}

The experimental results show that active thermography is a non-invasive, non-destructive measuring method to visualize initial subsurface defects in the millimeter range, which remain hidden during a visual inspection of the leading edge of wind turbine rotor blades. As a next step, the development of these initial material defects to premature rain erosion is investigated using computer tomography. With this knowledge, the in-situ defect measurement with thermography will also enable damage predictions for the rotor blade leading edge of real-scale wind turbines.

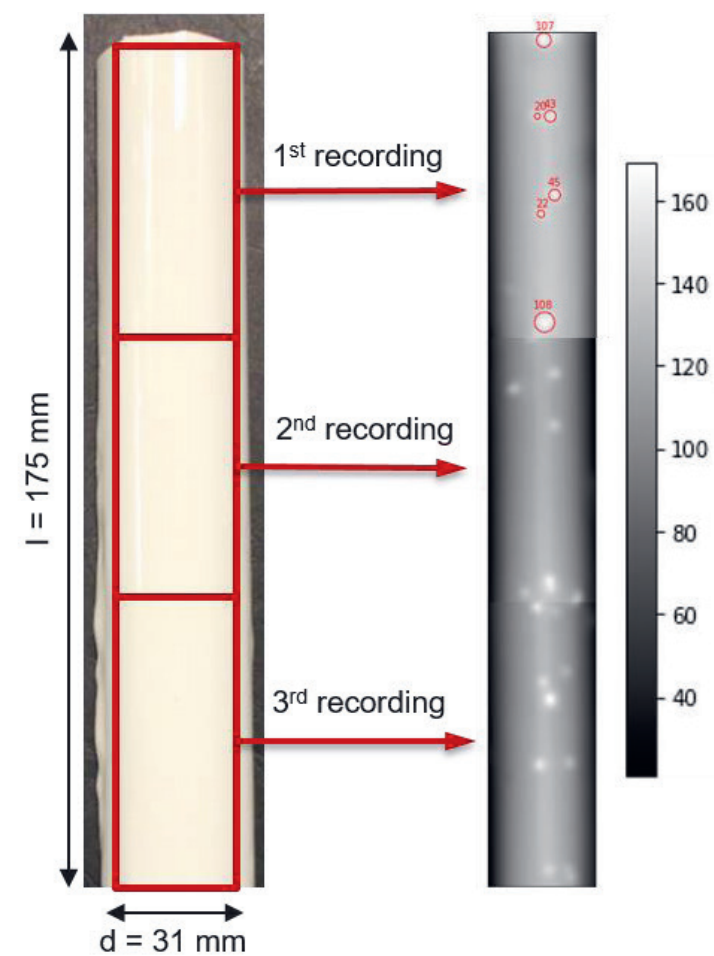

Fig. 3. Left: optical image of the modified test specimen; right: thermograms of the modified test specimen spread over three images - polystyrene balls are recognizable as bright points in the signal processed thermograms (marked with red circles and the corresponding CNR in the $1^{\text {st }}$ recording as an example)

Funding: German Federation of Industrial Research Association (AiF, 20794 N / 2)

\section{References}

[1] A. Sareen, C. A. SAP re, M. S. Selig, Effects of leading edge erosion on wind turbine blade performance, Wind Energy 17, 1531-1542 (2014); doi: 10.1002/we.1649

[2] M.H. Keegan, Wind Turbine Blade Leadeing Edge Erosion: An investigation of rain droplet and hailstone impact induced damage mechanisms, Ph.D. thesis (2014), University of Strathclyde, Glasgow

[3] Z. Wang, G. Tian, M. Mea, F. Ciampa, Image processing based quantitative damage evaluation in composites with long pulse thermography, NDT \& E International, 99 (2018); doi: 10.1016/j.ndteint.2018.07.004

[4] D. P. Almond, S. L. Angioni, S. G. Pickering, Long pulse excitation thermographic non-destructive evaluation, NDT \& E International, 87 (2017); doi: 10.1016/j.ndteint.2017.01.003

[5] C. Dollinger, N. Balaresque, M. Sorg, A. Fischer, IR thermographic visualization of flow separation in applications with low thermal contrast, Infrared Physics \& Technology, 88 (2018); doi: 10.1016/j.infrared.2017.12.001 